\title{
Shape-dependent universality in percolation
}

\author{
Robert M. Ziff ${ }^{\mathrm{a}, 1}$ Christian D. Lorenz ${ }^{\mathrm{a}, 2}$ Peter Kleban ${ }^{\mathrm{b}, 3}$ \\ ${ }^{a}$ Department of Chemical Engineering, University of Michigan, Ann Arbor, \\ Michigan 48109-2136 USA \\ ${ }^{\mathrm{b}}$ Laboratory for Surface Science and Technology and Department of Physics and \\ Astronomy, University of Maine, Orono, Maine 04469 USA
}

\begin{abstract}
The shape-dependent universality of the excess percolation cluster number and cross-configuration probability on a torus is discussed. Besides the aspect ratio of the torus, the universality class depends upon the twist in the periodic boundary conditions, which for example are generally introduced when triangular lattices are used in simulations.
\end{abstract}

\section{Introduction}

In standard random percolation, the universality of the critical exponents is quite familiar. The values of these universal quantities depend only upon the dimensionality of the system. This is also the case for the amplitude ratios of quantities such as the mean cluster size and correlation length that are defined essentially for infinite systems. Even though one might use finite systems to measure such quantities, in the limit of the system length scale $L \rightarrow \infty$, the shape of those finite systems becomes irrelevant as $L$ exceeds the correlation length $\xi(p)$.

However, if a system is simulated exactly at $p_{c}$ where $\xi$ is in principle infinite, or if one takes the scaling limit where $p$ approaches $p_{c}$ as $L$ is increased such that $\xi$ remains a fixed fraction of $L$, then the shape of the system (or the sequence of systems) becomes relevant. This leads to another and larger class of universal quantities, whose values depends upon the shape of the boundary

1 email: rziff@umich.edu

2 email: cdl@umich.edu

3 email: kleban@maine.edu

Preprint submitted to Elsevier Preprint 6 October 2018 
of the system and the boundary conditions, as well as the dimensionality. In spite of the resulting proliferation of classes, one still considers these quantities to be universal, because they remain independent of the "microscopic" features of the system (lattice type or continuum model used, whether site or bond percolation, etc.).

Examples of shape-dependent universal properties go back to the work of Privman and Fisher [1] concerning Ising models on a torus. In percolation, the various crossing problems considered by Langlands et al. [2,3], Aizenman [4], Cardy [5], Ziff [6], Hovi and Aharony [7], Hu et al. [8,9], Watts [10] and Pinson [11] show this type of universality. These crossing quantites are all defined in terms of macroscopic features of the system, so universality is clear. Recently, Aharony and Stauffer [12] have examined the shape dependence of critical universal ratios such as $L^{-d} S / P_{\infty}^{2}$ where $S$ is the mean size (in sites) of finite clusters and $P_{\infty}$ is the probability a site belongs to the "infinite" cluster (one spanning the system, by some consistent definition). Although thess quantities are defined on a microscopic (site) level, the microscopic aspect cancels out by scaling theory and the ratio is universal - but again system-shape dependent. Similar quantities for the Ising model were studied by Kamieniarz and Blöte [13] and Müller [14].

Here we discuss two shape-dependent universal quantities: the excess cluster number and the cross-configuration probability. We also focus on twisted boundaries and the subtleties of the triangular lattice.

\section{The excess number of clusters}

Exactly at the critical threshold, the number of clusters per site or per unit area is a finite non-universal constant $n_{c}$, whose values for site $(\mathrm{S})$ and bond (B) percolation on square (SQ) and triangular (TR) lattices were examined in detail in [15]. Here we quote those results in terms of the number per unit area, taking the lattice bond length to be unity: $n_{c}^{S-S Q}=0.0275981(3)$ and $n_{c}^{S-T R}=0.0203522(6)$. For B-SQ, Temperley and Lieb [16] showed

$$
n_{c}^{B-S Q}=\left[\left(-\frac{\cot \mu}{2} \frac{\mathrm{d}}{\mathrm{d} \mu}\right)\left\{\frac{1}{4 \mu} \int_{-\infty}^{\infty} \operatorname{sech}\left(\frac{\pi x}{2 \mu}\right) \ln \left(\frac{\cosh x-\cos 2 x}{\cosh x-1}\right) \mathrm{dx}\right\}\right]_{\mu=\frac{\pi}{3}}
$$

which evaluates simply to [15]

$$
n_{c}^{B-S Q}=\frac{3 \sqrt{3}-5}{2}=0.098076211 \ldots
$$


Likewise, Baxter, Temperley, and Ashley's [17] integral expression for $n_{c}^{B-T R}$ evaluates to $\left(35 / 6-2 / p_{c}^{B-T R}\right) \sqrt{3}=0.129146645 \ldots$ on a per-unit-area basis, where $p_{c}^{B-T R}=2 \sin (\pi / 18)$. Here, clusters in bond percolation are characterized by the number of wet sites they contain, with isolated sites corresponding to clusters of size one.

While $n_{c}$ is non-universal, its finite-size correction is universal. For an $L \times L^{\prime}$ critical system with periodic boundary conditions on all sides, the total number of clusters $N$ behaves as $N\left(L, L^{\prime}\right)=n_{c} L L^{\prime}+b+O\left(L L^{\prime}\right)^{-1}$ where $b$ is a universal function of $r=L^{\prime} / L[15]$. That is,

$$
b(r)=\lim _{L \rightarrow \infty}\left[N\left(L, L^{\prime}\right)-n_{c} L L^{\prime}\right]
$$

with $L^{\prime}=r L$, and $b$ represents the excess number of clusters over what one would expect from the bulk density. As such, it reflects the large clusters of the system, which is the basis of its universality. Aharony and Stauffer [12] showed that the universality of $b$ follows directly from the arguments of Privman and Fisher [1] applied to percolation, implying that $b$ is precisely the value of the free energy scaling function at $p_{c}$ and $h=0$. Note that this "free energy" is $\sum_{s} n_{s} e^{-h s}$, different from the free energy given below.

In [15], $b(1)$ was numerically found to equal 0.884 for both site and bond percolation on a SQ lattice, demonstrating universality. It was also found that $b(2)=0.991, b(\underset{\tilde{b}}{4})=1.512$, and for large $r$ (systems of very high aspect ratio), $b(r) \sim \tilde{b} r$ with $\tilde{b}=0.3608$. Here $\tilde{b}$ is the excess number per unit length along an infinite periodic strip or cylinder. Periodic b. c. are essential in this problem to eliminate boundary effects which would otherwise overwhelm $b$.

In [18] it was shown that $b$ can be found explicitly from exact coulomb-gas results. In the Fortuin-Kastelyn representation, the partition function of the Potts model at criticality is $Z=\sum Q^{N_{C}+N_{B} / 2}$ where $N_{C}$ is the number of clusters and $N_{B}$ the number of bonds, giving bond percolation in the limit of $Q=1$. Here the free energy is $F=\ln Z$, and $\left\langle N_{c}+N_{B} / 2\right\rangle$ follows from $d F / d Q$ at $Q=1$. Using the Potts model partition function (universal part) of Di Francesco et al. [19], [18] obtained

$$
\begin{gathered}
b(r)=\frac{5 \sqrt{3} r}{24}+q^{5 / 4}\left(2 \sqrt{3} r-\frac{1}{2}\right)+q^{2}(\sqrt{3} r-1)+q^{5 / 48}+2 q^{53 / 48} \\
-q^{23 / 16}+q^{77 / 48}+\ldots
\end{gathered}
$$

where $q=\mathrm{e}^{-2 \pi r}$. This result yields $b(1)=0.883576308 \ldots, b(2)=0.991781515 \ldots$ $b(4)=1.516324734 \ldots$, and $\tilde{b}=5 \sqrt{3} / 24$, consistent with measurements of [15]. The result for $\tilde{b}$ also follows directly from the work of Blöte, Cardy and 
Fig. 1. Two representations of a triangular lattice on a square array, yielding (a) a rectangular boundary of aspect ratio $\sqrt{3} / 2$ with no twist, and (b) the same rectangular boundary but with a twist of $1 / 2$.

Nightingale [20] on cylindrical systems. Fluctuations and higher-order cumulants are also discussed in [18], and $\tilde{b}$ in $3 \mathrm{~d}$ is discussed in [21].

\section{Confirmation of universality using triangular lattices}

Demonstrating universality of $b(r)$ by choosing only B-SQ and S-SQ as in [15] may not be completely convincing, and one would like to compare, say, a TR and SQ lattice. Periodic b. c. must be retained. Two obvious ways to represent the TR lattice on the square array of a computer program are shown in Fig 1. In (a), the TR lattice topology is created by choosing every other site of the SQ lattice. Taking an $L \times 2 L$ boundary on the SQ lattice as shown, the effective boundary on the TR lattice becomes a rectangle with $r=\sqrt{3} / 2$. Periodic $b$. c. on the underlying SQ lattice results in normal (untwisted) periodic b. c. on the TR lattice also. The measured $b=0.887$ for this system agrees completely with the theoretical $b(\sqrt{3} / 2)=0.887373266$ for a rectangular system with aspect ratio $\sqrt{3} / 2$.

The second obvious way to represent the TR lattice - by far the most common one - is shown in Fig. 1(b). Diagonals are simply added to the SQ lattice, and the periodic b. c. are applied to the squared-off lattice as is. Making this into a proper TR lattice, the system becomes a $1 \times 160^{\circ}$ rhombus, and shifting around the triangle as shown in Fig. 1b demonstrates that it is effectively a rectangular boundary with $r=\sqrt{3} / 2$, but with a "twist" $t=\frac{1}{2}$ in the periodic b. c., meaning that the $x$ coordinates are shifted by a fraction $t$ of the total length when wrapping around in the vertical direction. For this system, 
Fig. 2. Excess cluster number $b(r, t)$ vs. twist $t$ for $14 \times 16$ simulation (triangles), theory for $r=\sqrt{3} / 2$ (solid line), $16 \times 16$ simulation (squares) and theory for $r=1$ (broken line).

simulations gave $b(\sqrt{3} / 2,1 / 2)=0.878[15]$, less than $b(\sqrt{3} / 2,0)=0.8874$ and indeed less than the minimum untwisted rectangle, $b(1,0)=0.8836$, where now we write $b=b(r, t)$.

Here we demonstrate the universality of $b(\sqrt{3} / 2,1 / 2)$ by studying a SQ lattice with $r$ (necessarily rational) close to $\sqrt{3} / 2 \approx 0.866$, and comparing the results to the above TR lattice measurement. On the SQ lattice, we considered a system of size $14 \times 16$, where $r=0.875$; the measured values of $b$ for $t=0,1 / 8, \ldots, 1$ are shown in Fig. 2. At twist $\frac{1}{2}$, the value of $b$ is very close to the result 0.878 found on the TR lattice, [15] demonstrating the universality between these two lattices. Note that, to find $b(\sqrt{3} / 2,1 / 2)$ on the SQ lattice to high precision, one would have to consider different size systems and extrapolate to $\infty$, and different rational $r$ to interpolate to $r=\sqrt{3} / 2$. Results for a square system $16 \times 16$ are also shown in Fig. 2 .

We have generalized the theoretical methods described in [18] to find $b(r, t)$ from the partition function of [19]. The parameter $\tau$ becomes $t+i r$, and the results, which are rather involved, yield the solid curves in Fig. 2. The discrepancy with the numerical values can be attributed to the small system size of these simulations.

\section{Symmetries on a torus with a twist}

The torus with a twist has various topological symmetries that apply to any shape-dependent universal quantity $u(r, t)$, which includes $b(r, t)$. We consider 
a rectangular boundary with base 1 and height $r$, with a horizontal twist $t$ in the periodic b. c. (Note that having twists in two directions leads to a non-uniform system, so we don't consider it.) $u(r, t)$ satisfies the obvious symmetries of reflection

$$
u(r, t)=u(r,-t)
$$

and periodicity in the $t$ direction

$$
u(r, t)=u(r, 1+t)
$$

Another symmetry follows from the observation that the same rhombus can be made into a rectangle in two different ways, leading to:

$$
u(r, t)=u\left(\frac{r}{r^{2}+t^{2}}, \frac{t}{r^{2}+t^{2}}\right)
$$

Another construction shows that when $t=1 / n$ where $n$ is an integer,

$$
u\left(r, \frac{1}{n}\right)=u\left(\frac{1}{n^{2} r}, \frac{1}{n}\right)
$$

which also follows from Eqs. (5-7). On the complex $\tau=t+i r$ plane, (7) corresponds to $\tau \rightarrow 1 / \tau$ while (6) corresponds to $\tau \rightarrow \tau+1$. These transformations generate the modular group, and functions invariant under them are called modular. Thus, $b(r, t)$ must necessarily be a modular function. However, the explicit expression for $b$ does not display that modularity clearly.

Besides the excess number, another universal quantity on a torus is the crossconfiguration probability $\pi_{+}(r, t)$, which can be expressed in a quite compact form. Using the results of [19], Pinson [11] has shown $\pi_{+}(r, t)=\frac{1}{2}\left[Z_{c}(8 / 3)-\right.$ $\left.Z_{c}(2 / 3)\right]$, where

$$
Z_{c}(h)=\frac{\sqrt{h / r}}{\eta(q) \bar{\eta}(q)} \sum_{n, n^{\prime}} \exp \left\{-\frac{\pi h}{r}\left[n^{\prime 2}+n^{2}\left(r^{2}+t^{2}\right)-2 t n n^{\prime}\right]\right\},
$$

$\eta(q)$ is the Dedekind eta function and $q=e^{-2 \pi(r-i t)}$. It can be easily verified that this function satisfies the modular symmetries. For an untwisted torus (9) reduces to

$$
\pi_{+}(r, 0)=\frac{1}{4} \sqrt{\frac{3}{2}} \frac{\varphi\left(\frac{3 r}{8}\right) \varphi\left(\frac{3}{8 r}\right)-2 \varphi\left(\frac{3 r}{2}\right) \varphi\left(\frac{3}{2 r}\right)}{\tilde{\eta}(r) \tilde{\eta}\left(\frac{1}{r}\right)}
$$


where $\tilde{\eta}(r)=\eta\left(e^{-2 \pi r}\right)$ and $\varphi(r)=\vartheta_{3}\left(e^{-\pi r}\right)$ is the Jacobi theta function in Ramanujan's notation. The symmetry $r \rightarrow 1 / r$ is apparent. For all rational $r$, $\pi_{+}(r, 0)$ is an algebraic number; for example, for $r=1$, one can show

$$
\pi_{+}(1,0)=\frac{2 \sqrt{a+\bar{b}} \sqrt{2 \sqrt{a \bar{b}}}-(a+\bar{b})+2 \sqrt{a \bar{b}}}{3^{1 / 4} 4}=0.309526275 \ldots
$$

where $a=1+\sqrt{3}$ and $\bar{b}=\sqrt{2}-3^{1 / 4}$, using various results for theta functions. According to the symmetries above, this same value $\pi_{+}(1,0)=0.3095 \ldots$ applies to $(r, t)=(1 / 2,1 / 2)=(1 / 5,3 / 5)=(1 / 10,3 / 10)=(1 / 13,5 / 13)=$ $(1 / 17,13 / 17)=(1 / 26,5 / 26)=(1 / 29,12 / 29)=(1 / 34,13 / 34)=(1 / 37,6 / 37)=$ $(1 / 41,9 / 41)=(1 / 50,14 / 50)$ and infinitely many other systems, just as $b(1,0)=$ $0.8836 \ldots$ applies to all these systems.

We have also measured $\pi_{+}$in the $14 \times 16$ and $16 \times 16$ systems, with various $t$. Whether crossing occurs can be found using an indicator function, such as $I=N_{C}-N_{C^{\prime}}+N_{B}-N_{S}$, where $N_{C}$ is the number of clusters, $N_{C^{\prime}}$ is the number of dual-lattice clusters, $N_{B}$ is the number of bonds, and $N_{S}$ is the number of sites. When $I=1$, there is a cross-configuration on the lattice, when $I=-1$ there is a cross configuration on the dual-lattice (these two events are clearly mutually exclusive), and when $I=0$ there is neither. In the latter case, there will necessarily be at least one wrap-around cluster on the lattice or dual-lattice, or a spiral. Another indicator function can be made using the number of hulls in the system [11].

In Fig. 3 we show the measured $\pi_{+}(r, t)$ and comparison with predictions of Pinson's formula. The small deviations are presumably due to finite-size effects which should disappear when larger systems are measured and an extrapolation is made to infinity, as we have verified for $t=0$. Note that $\pi_{+}(\sqrt{3} / 2,1 / 2)=0.316053413 \ldots$ is at a local and apparently global maximum of Pinson's formula, although again by equations (5-7) an infinite number of other points on the $(r, t)$ plane have the identical value of $\pi_{+}$, such as $(\sqrt{3} / 6,1 / 2)$ and $(\sqrt{3} / 14,5 / 14)$.

A plot of Pinson's formula as a function of $t$ for different $r$ shows that for small $r$ it begins to develop oscillations. This is because of the tendency to create spiral rather than cross configurations for small aspect ratios.

\section{The meaning of $b$}

In [15], it was suggested that $b$ relates to the number of "spanning" clus-

ters, since these are essentially the cause of the excess. However, Hu [22] has 
Fig. 3. Pinson's number $\pi_{+}(r, t)$ vs. twist $t$; legend same as Fig. 2.

shown that they are not numerically identical, using one particular definition of spanning. Here we elaborate on this point.

We consider large systems with periodic b. c. and ask for the number of clusters $\operatorname{Nr}\left(\ell_{m} \geq \ell\right)$ whose maximum dimension $\ell_{m}$ in the $x$ or $y$ direction exceeds some value $\ell$. Since $s \sim \ell_{m}^{D}$ where $D$ is the fractal dimension and $n_{s} \sim s^{-\tau}$, it follows that $\operatorname{Nr}\left(\ell_{m} \geq \ell\right) \sim \ell^{(1-\tau) D}=\ell^{-d}$ or $\ell^{-2}$ in $2 \mathrm{~d}$. We have measured this quantity for square $L \times L$ and rectangular $L \times 2 L$ systems for various sizes, and find that for an intermediate range in $\ell$ the expected universal $\ell^{-2}$ behavior is followed:

$$
\operatorname{Nr}\left(\ell_{m} \geq \ell\right)=C\left(\frac{\ell}{\sqrt{A}}\right)^{-2}=C A / \ell^{2}
$$

where $A=L^{2}$ (square) or $2 L^{2}$ (rectangular system) and $C=0.116 . \quad C$ is a universal measure of the size distribution, dependent only upon the rule of what constitutes the length scale $\ell$. (One could just as well use maximum diameter, radius of gyration, etc., each of which would lead to a different $C$. Thus $C$ too is a "shape"-dependent universal quantity.) Eq. (12) implies that $C$ is just the density in an infinite system of clusters of minimum dimension $\ell$, on the length scale of $\ell$. It is the universal analog of $n_{c}$.

The data for $\operatorname{Nr}\left(\ell_{m} \geq \ell\right)$ deviates from Eq. (12) at both the large and small size limits. For small $\ell$, the deviation is due to lattice-level effects; at the limit $\ell=1, \operatorname{Nr}\left(\ell_{m} \geq 1\right)$ is just $n_{c} A$, which is clearly non-universal. For $\ell$ near the maximum, the deviation is due to the influence of the boundary and is related to the value of $b$.

According to our definition, $b=$ (the actual number of clusters) - (the expected number of clusters using the bulk density). Therefore, using a lower 
length scale of $\ell$ that is in the scaling region, we have

$$
b=\operatorname{Nr}\left(\ell_{m} \geq \ell\right)-C A / \ell^{2}
$$

Evidently, in using this formula, $\ell$ can be taken right up to the minimum dimension of the system, $L$. We find that the number in each size range, $N_{\ell}=\operatorname{Nr}\left(\ell / 2 \leq \ell_{m}<\ell\right)=\operatorname{Nr}\left(\ell_{m} \geq \ell / 2\right)-\operatorname{Nr}\left(\ell_{m} \geq \ell\right)$ follows $N_{\ell / 2} / N_{\ell}=4$ within \pm 0.01 right up to $\ell=L$. This implies that $b$ can be found by applying (13) with $\ell=L$. Using the numerical data for $\operatorname{Nr}\left(\ell_{m} \geq L\right)$ for $r=1$ and $r=2$, we find from (13)

$$
\begin{aligned}
& b(1,0)=0.990-0.116=0.87 \\
& b(2,0)=1.214-2(0.116)=0.98
\end{aligned}
$$

compared with the actual vales 0.884 and 0.991 respectively. The small shortfall may be due to the need to take $\ell$ somewhat smaller than $L$ in (13), or to statistical errors. We are investigating this point further.

Thus, we have the meaning of $b$ (taking $\ell=L$ ): the number of clusters in a system of area $A$ whose extent is larger than the minimum system dimension $L$, minus the expected number predicted by the bulk density, $C A / L^{2}$.

\section{Conclusions}

We have numerically demonstrated the universality of $b(\sqrt{3} / 2,1 / 2)$ on both the SQ and TR lattices. We have shown that $b$ is related to the average number of clusters of length scale greater or equal to $L$, if one subtracts off the contribution of the universal size distribution characterized by the universal constant $C$.

It appears that $N_{\ell}$ follows a universal behavior close to, and perhaps right up to $\ell=L$. On a finite system with periodic b. c., the probability of growing a cluster of a certain size is identical to its probability on an infinite system, as long as the cluster is small enough that it doesn't touch itself after wrapping around the boundaries. This result, however, says that the number of clusters of a certain size range is also substantially unaffected by the system finiteness, even though the boundaries should, it seems, influence the statistics of such clusters when their combined size is large enough that they touch when wrapping around the boundary. Further work needs to be done to understand this behavior.

The TR lattice constructed as in Fig. $1 \mathrm{~b}$ gives extremal values of $b$ and $\pi_{+}$and 
may in fact be the best periodic system to use for many percolation problems (as well as other types of lattice simulations). This is because, when the $1 \times 1$ $60^{\circ}$ rhombus is used to tile the plane, it leads to a triangular array of repeated patterns which has the most space between each repeated element of any regular array.

While tori with "twists" add a rich extra degree of freedom, they are by no means the only systems for which $b$ can be calculated. What is needed is a system that is effectively a closed surface. One can transform the rectangular basis of the torus to other shapes by conformal transformation, such as to an annulus, and then apply the transformed periodic b. c. to the problem. (Here, the curved boundaries suggest using a continuum form of percolation, as in [23]). A simple closed surface like the surface of a sphere can also be used for the system. Each of these systems will have its own characteristic value of $b$ and other shape-dependent universal quantities.

\section{Acknowledgements}

RZ acknowledges NSF grant DMR-9520700. Correspondence with K. S. Williams and B. Berndt concerning theta function identities is gratefully acknowledged.

\section{References}

[1] V. Privman and M. E. Fisher, Phys. Rev. B 30, 322 (1984).

[2] R. P. Langlands, C. Pichet, P. Pouliot, and Y. Saint-Aubin, J. Stat. Phys. 67, 553 (1992).

[3] R. P. Langlands, P. Pouiliot, and Y. Saint-Aubin, Bull. AMS 30, 1 (1994).

[4] M. Aizenman, in The IMA Volumes in Mathematics and its Applications (Springer-Verlag, 1997).

[5] J. L. Cardy, J. Phys. A: Math Gen. 25, L201 (1992).

[6] R. M. Ziff, Phys. Rev. Lett.69 2670 (1992).

[7] J.-P. Hovi and A. Aharony, Phys. Rev. E 53, 235 (1996).

[8] C.-K. Hu and C.-Y. Lin, Phys. Rev. Lett. 75, 193 (1995).

[9] C.-K. Hu, C.-Y. Lin and J.-A. Chen, Phys. Rev. Lett. 77, 8 (1996).

[10] G. M. T. Watts, J. Phys. A: Math Gen. 29, L363 (1996).

[11] H. T. Pinson, J. Stat. Phys. 75, 1167 (1994). 
[12] A. Aharony and D. Stauffer, J. Phys. A: Math Gen. 30, L301 (1997).

[13] G. Kamieniarz and H. W. J. Blöte, J. Phys. A 26, 201 (1993).

[14] B. Müller, Int. J. Mod. Phys. C 9, 1 (1998).

[15] R. M. Ziff, S. R. Finch, and V. S. Adamchik, Phys. Rev. Lett., 793447 (1997).

[16] H. N. V. Temperley and E. H. Lieb, Proc. R. Soc. Lond. A. 322, 251 (1971).

[17] R. J. Baxter, H. N. V. Temperley, and S. E. Ashley, Proc. R. Soc. Lond. A. 358, 535 (1978).

[18] P. Kleban and R. M. Ziff, Phys Rev. B 57, R8075 (1998).

[19] P. Di Francesco, H. Saleur, and J. Zuber, J. Stat. Phys. 49, 57 (1987).

[20] H. Blöte, J. Cardy, and M. Nightingale, Phys. Rev. Lett.56 742 (1986).

[21] C. D. Lorenz and R.M. Ziff, Phys. Rev. E 57, 230 (1998).

[22] C.-K. Hu, Rutgers Statistical Physics Meeting, December 1997.

[23] H.-P. Hsu, M.-C. Huang, and K.-J. Ling, Phys. Rev. B 56, 10743 (1997). 

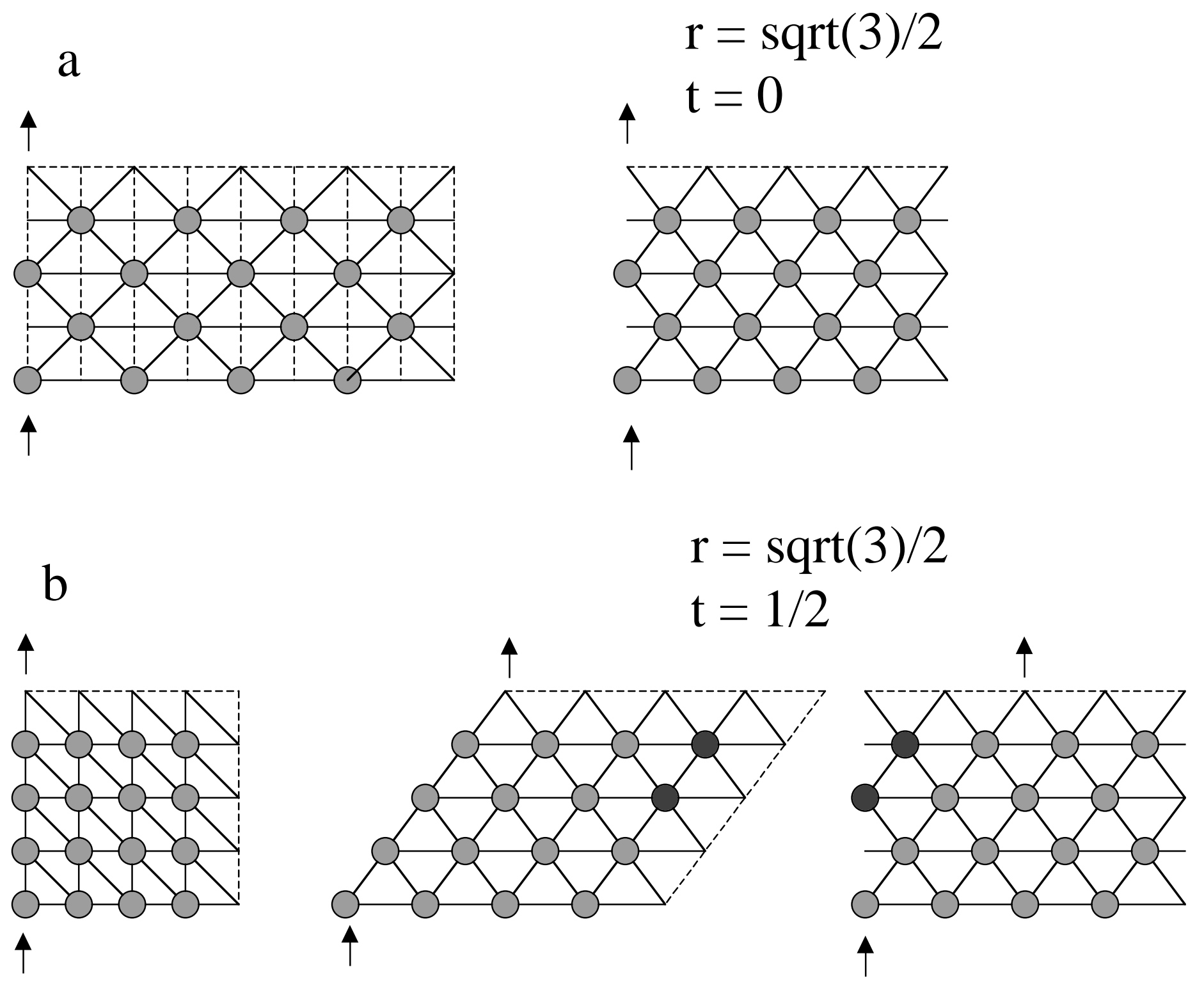
Ziff, Lorenz and Kleban, Fig. 2

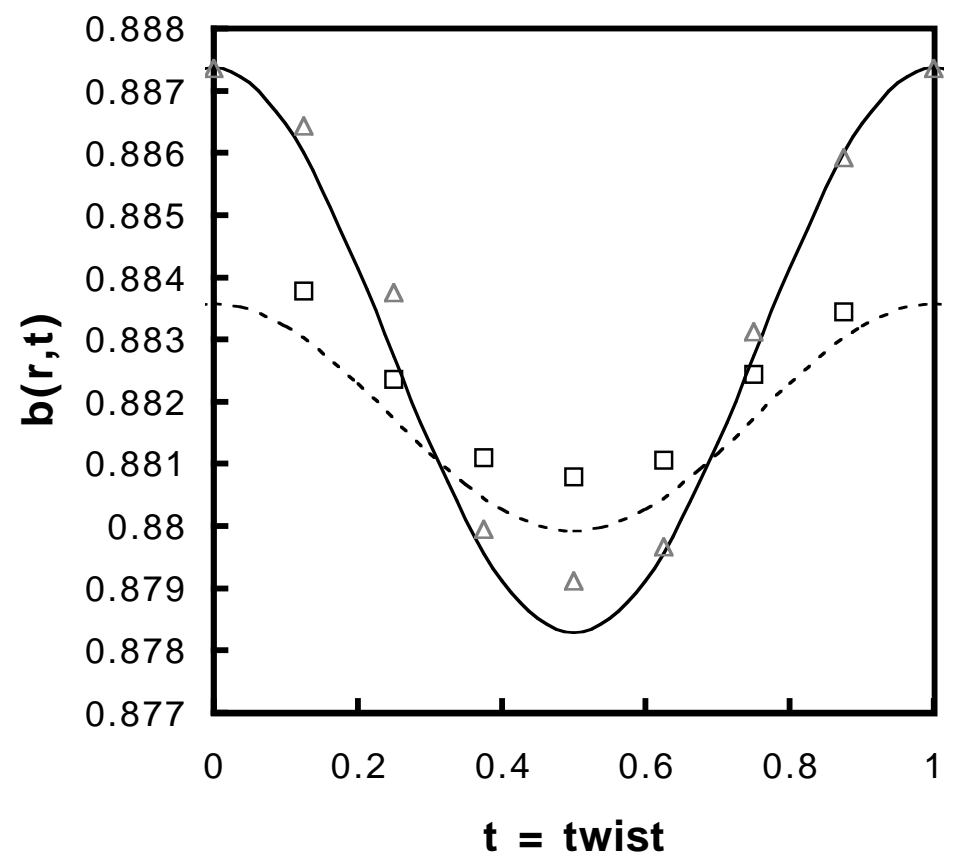


Ziff, Lorenz and Kleban, Fig. 3

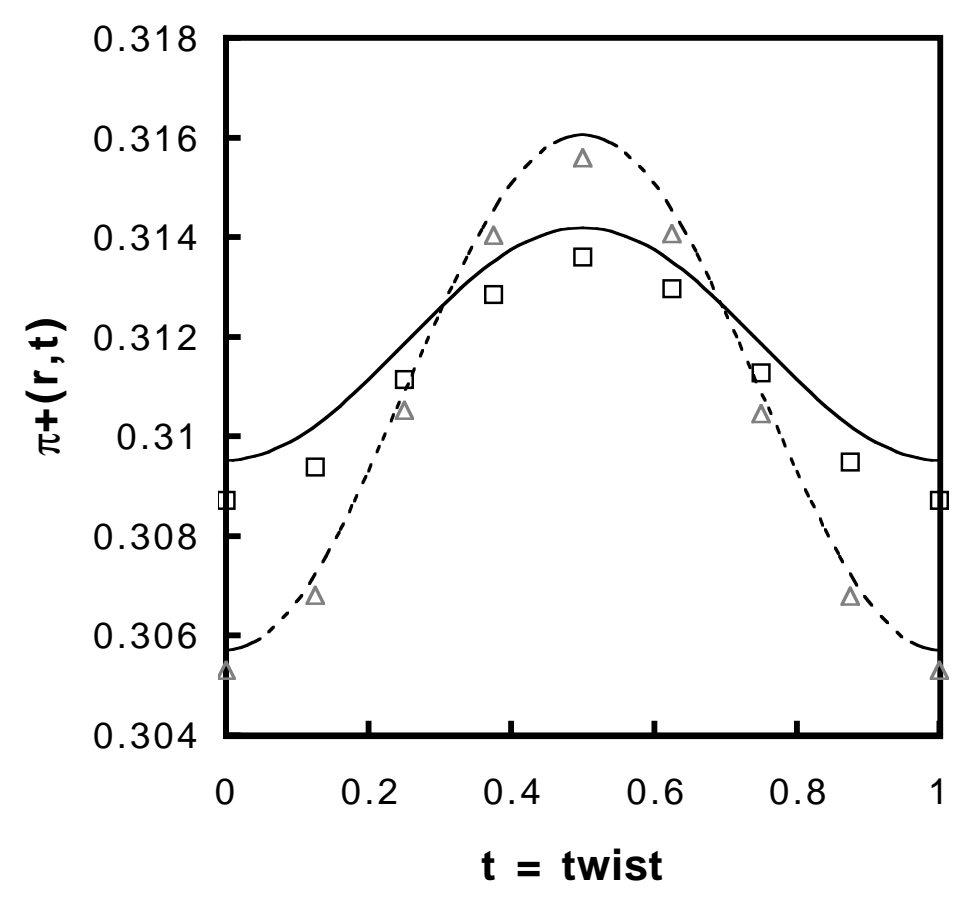

